\title{
Structural and functional
}

\section{consequences of succinate dehydrogenase subunit $B$ mutations}

\author{
E Kim 1, ${ }^{1,}$, E M Rath ${ }^{3, *}$, V H M Tsang ${ }^{1,2, *}$, A P Duff ${ }^{4}$, B G Robinson ${ }^{1,2}$, W B Church ${ }^{3}$, \\ D E Benn', T Dwight ${ }^{1}$ and R J Clifton-Bligh ${ }^{1,2}$
}

${ }^{1}$ Cancer Genetics, Kolling Institute of Medical Research, Royal North Shore Hospital, and University of Sydney, Sydney, New South Wales, Australia

${ }^{2}$ Department of Endocrinology, Royal North Shore Hospital, Sydney, New South Wales, Australia

${ }^{3}$ Faculty of Pharmacy, University of Sydney, Sydney, New South Wales, Australia

${ }^{4}$ Australian Nuclear Science and Technology Organisation, Lucas Heights, New South Wales, Australia

*(E Kim, E M Rath and V H M Tsang contributed equally to this work)

\author{
Correspondence \\ should be addressed \\ to E Kim \\ Email \\ edward.kim@sydney.edu.au
}

\begin{abstract}
Mitochondrial dysfunction, due to mutations of the gene encoding succinate dehydrogenase (SDH), has been implicated in the development of adrenal phaeochromocytomas, sympathetic and parasympathetic paragangliomas, renal cell carcinomas, gastrointestinal stromal tumours and more recently pituitary tumours. Underlying mechanisms behind germline SDH subunit B (SDHB) mutations and their associated risk of disease are not clear. To investigate genotype-phenotype correlation of SDH subunit B (SDHB) variants, a homology model for human $S D H$ was developed from a crystallographic structure. $S D H B$ mutations were mapped, and biochemical effects of these mutations were predicted in silico. Results of structural modelling indicated that many mutations within SDHB are predicted to cause either failure of functional SDHB expression (p.Arg27*, p.Arg90*, c.88delC and c.311delAinsGG), or disruption of the electron path (p.Cys101Tyr, p.Pro197Arg and p.Arg242His). GFP-tagged WT SDHB and mutant SDHB constructs were transfected (HEK293) to determine biological outcomes of these mutants in vitro. According to in silico predictions, specific $S D H B$ mutations resulted in impaired mitochondrial localisation and/or SDH enzymatic activity. These results indicated strong genotype-functional correlation for $S D H B$ variants. This study reveals new insights into the effects of $S D H B$ mutations and the power of structural modelling in predicting biological consequences. We predict that our functional assessment of $S D H B$ mutations will serve to better define specific consequences for SDH activity as well as to provide a much needed assay to distinguish pathogenic mutations from benign variants.
\end{abstract}

\author{
Key Words \\ - succinate dehydrogenase \\ - SDHB \\ - phaeochromocytoma \\ - paraganglioma \\ - functional consequences
}

Endocrine-Related Cancer (2015) 22, 387-397

\section{Introduction}

Phaeochromocytoma (PC) and paraganglioma (PGL) are rare neuroendocrine tumours that arise from adrenal glands and extra-adrenal sites respectively (Amar et al.
2005, Gimenez-Roqueplo et al. 2012). These tumours occur in the context of inherited neoplasia syndromes in more than $30 \%$ of cases and are linked to germline

Published by Bioscientifica Ltd 
mutations in succinate dehydrogenase subunit A ( $S D H A)$, SDHB, SDHC, SDHD, SDHAF2, VHL, RET, NF1, TMEM127, MAX, KIF1B, EPAS1 (Lorenzo et al. 2013), FH (Castro-Vega et al. 2014 and reviewed by Dahia (2014)) and MDH2 (Poliakov et al. 2014). Mutations in any of the subunits of $S D H$, also known as complex II of the mitochondrial respiratory chain (MCII), have been associated with development of PC/PGL and also renal cell cancers, gastrointestinal stromal tumours and pituitary adenomas (Dahia et al. 2005, Dwight et al. 2013, Gill et al. 2010, 2011). Mutations in $S D H B$ are the most commonly found gene mutations in PC/PGL and are associated with younger ages at presentation, higher rates of metastases and poorer prognosis (Srirangalingam et al. 2008). Screening programmes have been recommended for surveillance of PC/PGL and the associated neoplasms in subjects carrying $S D H x$ mutations (Lenders et al. 2014); however, penetrance of disease is highly variable, which therefore burdens many asymptomatic individuals with lifetime screening for low-risk events (Benn et al. 2006). Genotype-phenotype correlations have been difficult to establish, although the precise mechanism(s) by which these mutations cause defective SDH function has been under-studied to date. SDH is located in the inner mitochondrial membrane and matrix and consists of four subunits (A, B, C and D), which function both in the Krebs cycle and as part of the respiratory chain. In the respiratory chain, MCII functions to transport electrons to the ubiquinone pool, then to cytochrome $c$ of complex III. In the Krebs cycle, SDH catalyses the conversion of succinate to fumarate (Imperiale et al. 2013). The SDH structure choreographs the transfer of electrons by catalysing a twoelectron reduction of succinate to fumarate via a twoelectron reduction of its flavin adenine dinucleotide (FAD) cofactor, finally producing two stepwise single-electron transfers to ubiquinone to reduce it to ubiquinol (Van Vranken et al. 2014). Two predictable consequences therefore of SDH inactivation and malfunction are accumulation of succinate and increased production of reactive oxygen species. Both outcomes may contribute to cellular accumulation of hypoxia-inducible factors (HIFs; Denko 2008, Jochmanová et al. 2014). The results of various yeast model studies (Barrientos 2003, Goffrini et al. 2009, Panizza et al. 2013) have indicated that selective missense germline mutations in SDHB can lead to significantly reduced SDH enzyme activity. Results of another recent study have indicated that $S D H B$ mutations are often associated with impaired mRNA expression and reduced protein stability (Yang et al. 2012). In silico modelling of the tetrameric SDH enzyme complex from crystallographic data has tentatively predicted a broad spectrum of biochemical consequences of SDHB mutations (Sun et al. 2005).

The aim of this study was to carefully assess the functional consequences of disease-associated $S D H B$ mutations using in silico modelling of SDH followed by two biological assays, to assess the effects of SDHB mutations on mitochondrial localisation, SDH activity and interaction with SDHA. We sought to address whether different $S D H B$ mutations cause distinct alterations in protein function, thereby providing a platform for more informative genotype-phenotype associations in the future.

\section{Materials and methods}

\section{Structural modelling}

We created a homology model of human SDH by mapping the human protein sequences onto a template structure in such a way as to respect the biophysical properties and interactions of the residues observed in the template. The template structure used was pig heart SDH, PDBID 1ZOY (Sun et al. 2005, resolution 2.40 ̊) from the Protein Databank (wwPDB, Berman et al. 2000), which was the closest structurally determined homologue to human $\mathrm{SDH}$. The homology model was created because a highresolution experimentally determined structure of human $\mathrm{SDH}$ is not currently available. The Entrez protein database sequences from which the human SDH homology model was built were those of the human SDH genes NP_004159, NP_002991, NP_002992 and NP_002993, for SDHA, SDHB, SDHC and SDHD, respectively, and had sequence identities of $96,98,91$ and $94 \%$, respectively, to the pig heart sequences. This WT human SDH model was then used as the template model to build homology models for mutant human SDH proteins. Our choice of $S D H B$ mutations for in silico structural modelling was principally based upon their association, in our experience, with severe clinical disease (i.e. young age of onset and/or malignancy; Table 1). Several additional mutations were selected from the Leiden Open Variations Database (LOVD; Fokkema et al. 2011), together with singlenucleotide polymorphisms in the LOVD catalogued as not associated with disease (Supplementary Tables 1 and 2, see section on supplementary data given at the end of this article). Overall, our panel of $S D H B$ mutants was representative of truncating mutations, nonsense mutations and missense mutations spread throughout the exons. Two software modules, Sorting Intolerant From Tolerant (SIFT; http://sift.jcvi.org/; Ng \& Henikoff 2001) and

Published by Bioscientifica Ltd. 
Table 1 Predicted consequences of SDHB mutations based on structural modelling or in silico tools

\begin{tabular}{|c|c|c|c|c|c|}
\hline \multirow[b]{2}{*}{$S D H B^{a}$ mutation } & \multirow[b]{2}{*}{ Predicted structural consequence $^{b}$} & \multirow{2}{*}{$\begin{array}{l}\text { Earliest age } \\
\text { of onset } \\
\text { (years) }\end{array}$} & \multirow{2}{*}{$\begin{array}{l}\text { Phenotype in } \\
\text { families }\end{array}$} & \multicolumn{2}{|c|}{ In silico } \\
\hline & & & & SIFT & Polyphen-2 \\
\hline p.Arg27* & $\begin{array}{l}\text { Truncation mutation - missing protein chain abrogates } \\
\text { SDH assembly }\end{array}$ & $15^{A, C}$ & EA & - & - \\
\hline p.Ala43Pro & $\begin{array}{l}\text { Mutation having no obvious consequence and located } \\
\text { in a disease-associated mutation hot-spot - peripheral } \\
\text { residue may compromise assembly factor binding }\end{array}$ & $27^{B, C}$ & $\mathrm{EA}, \mathrm{M}$ & $\mathrm{D}(0.03)$ & B (0.356) \\
\hline p.Arg46Gly & $\begin{array}{l}\text { Mutation having no obvious consequence and located } \\
\text { in a disease-associated mutation hot-spot - peripheral } \\
\text { residue may compromise assembly factor binding }\end{array}$ & $29^{B, C}$ & $E A, H N, M, P C$ & $D(0.00)$ & $\mathrm{D}(1.0)$ \\
\hline p.Arg46Gln & $\begin{array}{l}\text { Mutation having no obvious consequence and located } \\
\text { in a disease-associated mutation hot-spot - peripheral } \\
\text { residue may compromise assembly factor binding }\end{array}$ & $23^{B}$ & $E A, H N, M, P C$ & $D(0.00)$ & $D(1.0)$ \\
\hline p.Arg90* & $\begin{array}{l}\text { Truncation mutation - missing protein chain abrogates } \\
\text { SDH assembly }\end{array}$ & $13^{\mathrm{A}}$ & EA, HN & - & - \\
\hline p.Cys101Tyr & $\begin{array}{l}\text { Mutation breaking electron pathways - due to loss of } \\
\text { iron-sulphur centre }\end{array}$ & $10^{c}$ & EA & $\mathrm{T}(0.10)$ & $D(1.0)$ \\
\hline p.lle127Ser & $\begin{array}{l}\text { Mutation having no obvious consequence and located } \\
\text { in a disease-associated mutation hot-spot - non- } \\
\text { peripheral residue may compromise assembly } \\
\text { factor binding }\end{array}$ & $18^{\mathrm{B}}$ & $E A, M$ & $\mathrm{D}(0.00)$ & $\mathrm{D}(0.99)$ \\
\hline p.Pro197Arg & $\begin{array}{l}\text { Mutation affecting electron pathways - may result in } \\
\text { loss of electrons and generation of reactive oxygen } \\
\text { species }\end{array}$ & $16^{\mathrm{B}, \mathrm{C}}$ & EA, PC & $\mathrm{D}(0.01)$ & $\mathrm{D}(0.999)$ \\
\hline p.Arg $242 \mathrm{His}$ & $\begin{array}{l}\text { Mutation disrupting macromolecular assembly - } \\
\text { compromises SDHD assembly }\end{array}$ & $19^{\mathrm{A}}$ & EA & $\mathrm{D}(0.00)$ & $D(1.0)$ \\
\hline c.88delC & Model not created for this frameshift mutation & $13^{\mathrm{A}}$ & $\mathrm{M}, \mathrm{PC}, \mathrm{RCC}$ & - & - \\
\hline c.311delAinsGG & Model not created for this frameshift mutation & $27^{A}$ & EA, HN, PC, RCC & - & - \\
\hline
\end{tabular}

$\mathrm{SDH}$, succinate dehydrogenase; SDHB, SDH subunit $\mathrm{B} ; \mathrm{PC}$, adrenal phaeochromocytoma; EA, extra-adrenal paraganglioma; HN, head and neck paraganglioma; M, malignancy; RCC, renal cell carcinoma; $\mathrm{B}$, benign; $\mathrm{D}$, damaging; T, tolerated; SIFT, Sorting Intolerant From Tolerant; Polyphen-2, Polymorphism Phenotyping-2. Source of Clinical Information: A Australian SDH Consortium (DE Benn, T Dwight, K Tucker and R Clifton-Bligh unpublished data); ${ }^{B}$ International SDH Consortium (Benn et al. 2006) and CLeiden Database (Bayley et al. 2005).

a SDHB reference sequence: NM_003000 (mRNA) and NP_002991 (protein).

${ }^{b}$ Predictions for the human SDH homology model (based on pig heart SDH, PDBID 1ZOY (Sun et al. 2005)).

Polymorphism Phenotyping-2 (Polyphen-2; http:// genetics.bwh.harvard.edu/pph2/; Adzhubei et al. 2010), were used to predict pathogenicity of $S D H B$ mutants. All homology models were built using the Modeller Software (Šali \& Blundell 1993, Eswar et al. 2006) version 9.8 downloaded from http://salilab.org/modeller/, using default parameters and the molecular dynamics feature for refining residue positions. Hydrogen bonds in the models were calculated by HBPLUS (McDonald \& Thornton 1994) with the LIGPLOT Software (Wallace et al. 1995). The PyMOL Software (DeLano 2002) was used to visually inspect homology models. The Pathways Software (Beratan et al. 1991, Balabin et al. 2012) run in the VMD Software (Humphrey et al. 1996) was used to calculate the electronic coupling of segments of the electron path for the WT SDH model and for mutant models having a mutation between redox centres. Peptide bonds and metal bonds were treated as covalent bonds for the purposes of electron tunnelling mediation calculations.

\section{Generation of SDHB expression vectors}

A primer was designed to generate the WT SDHB in pEGFPN1 (Clontech) vector: AATGGAATTCTAAAAATGGCGGCGGTGGTCGCACTC. GFP vector and $S D H B$ insert were digested using EcoR1 and BamHI (Fermentas, Waltham, MA, USA). JM109 competent cells (Promega) were added to ligation reactions of empty vector and $S D H B$ insert that were then incubated on agar plates at $37^{\circ} \mathrm{C}$ overnight. Colonies from agar plates were amplified using PCR and then sequenced for confirmation. Primers were prepared (Supplementary Tables 3, 4 and 5, see section on supplementary data given at the end of this article) in order to perform site-directed mutagenesis according to the manufacturer's instructions using the Quickchange Lightening Site-Directed Mutagenesis Kit (Agilent Stratagene, Santa Clara, CA, USA) to generate SDHB mutants (p.Arg27*, p.Ala43Pro, p.Arg46Gly, p.Arg46Gln, p.Arg90*, p.Cys101Tyr, p.Ile127Ser, p.Pro197Arg, p.Arg242His,

Published by Bioscientifica Ltd 
c.88delC and c.311delAinsGG). Sanger sequencing was performed on the amplified products to confirm the presence of the mutation.

\section{Cell culture and transfection}

Human embryonic kidney 293 (HEK293) cells (American Type Culture Collection (ATCC), Manassas, VA, USA) were cultured in DMEM (Life Technologies) with 10\% fetal bovine serum (Life Technologies) at $37{ }^{\circ} \mathrm{C}$ and $5 \% \mathrm{CO}_{2}$. Rat PC cells (PC12 and ATCC) were cultured in DMEM (Life Technologies) supplemented with $10 \%$ fetal bovine serum (Life Technologies) and 5\% horse serum (Life Technologies). Cells (passages 15-23) were plated at a density of $1.0 \times 10^{6}$ cells $/ 25 \mathrm{~cm}^{2}$ flasks and allowed to settle overnight. WT $S D H B$, mutant $S D H B$ and empty vector DNA $(7.5 \mu \mathrm{g})$ were mixed with $15 \mu$ l Lipofectamine 2000 (Life Technologies) and then at room temperature for $15 \mathrm{~min}$ in $650 \mu \mathrm{l}$ Opti-MEM (Life Technologies). The mixtures were then used to transfect cells with replenished serum-free DMEM for $24 \mathrm{~h}$ (incubated at $37^{\circ} \mathrm{C}$ and $5 \%$ $\mathrm{CO}_{2}$ ). The cells were washed with PBS and lysed using either a subcellular fractioning kit or immunoprecipitation (IP) lysis buffer.

\section{Subcellular localisation}

Membrane fractions containing mitochondrial proteins were extracted using a Qproteome cell compartment kit according to the manufacturer's instructions (Qiagen). Membrane protein lysate was homogenised by brief sonication, and protein concentration was determined using the BCA Protein Assay Kit (Pierce Biotechnology, Rockford, IL, USA).

\section{Immunoprecipitation}

Dynabeads M-280 sheep anti-mouse IgG (Life Technologies) was incubated with either a mouse IgG antibody (dilution 1:2000, Thermo-Fisher, Waltham, MA, USA) for negative control or a GFP monoclonal mouse antibody (dilution 1:2000, Roche) for $2 \mathrm{~h}$ before washing, then it was incubated overnight with cell extracts in IP lysis buffer (20 mM Tris pH 7.5, $150 \mathrm{mM} \mathrm{NaCl}, 1 \mathrm{mM}$ EGTA, $1 \mathrm{mM}$ EDTA, and $0.1 \%$ Triton $\mathrm{X}-100$ ) at $4{ }^{\circ} \mathrm{C}$ under gentle rotation. Proteins not associated with GFP-tagged SDHB were removed $(3 \times 10 \mathrm{~min}$ gentle agitation washes) from the beads using IP lysis buffer with a higher salt concentration ( $500 \mathrm{mM} \mathrm{NaCl})$. Fresh IP lysis buffer was then re-introduced to the beads to prevent drying and degradation.

\section{Western immunoblotting}

Cell lysates were sonicated, mixed with NuPAGE LDS sample buffer and dithiothreitol and then separated by SDS-PAGE (4-12\% NuPAGE Bis-Tris gels, Invitrogen) under reducing conditions. Proteins were transferred to nitrocellulose membranes and the membrane blocked with 5\% skim milk (in TBST) for $1 \mathrm{~h}$ at room temperature. The membranes were probed with the following antibodies: GFP (dilution 1:2000, Roche (11814460001)), SDHA (dilution 1:1000, Abcam (ab14715), Cambridge, UK), MT-CO2 (dilution 1:2000, Abcam (ab3298)) and GAPDH (dilution 1:5000, Cell Signaling (D16H11), Danvers, MA, USA), and incubated overnight at $4{ }^{\circ} \mathrm{C}$. Immunoblots were washed three times with TBST for 5-10 $\mathrm{min}$ and incubated with the relevant secondary antibody conjugated to HRP. Blots were then washed (three times in TBST for $5 \mathrm{~min}$ ) and protein detected (ECL Plus Western Blotting Detection Reagent (GE Healthcare, Little Chalfont, UK)) on a LAS-3000 (Fujifilm, Brookvale, NSW, Australia). Quantitation was performed using the Multi-Gauge 3.11 Software (Fujifilm).

\section{SDH activity assay}

The decrease in absorbance due to reduction of the artificial electron acceptor dichlorophenolindophenol (DCPIP) linked to succinate was measured as SDH converting succinate to fumarate. An assay agent containing $25 \mathrm{mM}$ potassium phosphate buffer, $5 \mathrm{mM}$ $\mathrm{MgCl}_{2}, \mathrm{pH}$ 7.2, $20 \mathrm{mM}$ sodium succinate, $50 \mu \mathrm{M}$ DCPIP, $2 \mathrm{mM} \mathrm{KCN}, 2 \mu \mathrm{g} / \mathrm{ml}$ antimycin $\mathrm{A}, 2 \mu \mathrm{g} / \mathrm{ml}$ rotenone (in ethanol) and water was prepared for $500 \mu \mathrm{l}$ reaction volume/sample. The reaction mixture was incubated at $30{ }^{\circ} \mathrm{C}$ for $10 \mathrm{~min}$ to equilibrate, and then pre-incubated with GFP IP beads for $10 \mathrm{~min}$ at $30^{\circ} \mathrm{C}$ (Kirby et al. 2007). The reaction mixture was separated from the magnetic bead and placed into a 48-well plate, with baseline absorbance measured at a wavelength of $600 \mathrm{~nm}$ for 3 min before being mixed with beads again. An additional $6 \mu \mathrm{l}$ of $5 \mathrm{mM}$ DCPIP was then added to the bead/reaction mixture to initiate the enzyme-catalysed reaction, with reduction of DCPIP measured by decrease in absorption at $600 \mathrm{~nm}$ (extinction coefficient of $19.1 / \mathrm{mM}$ per $\mathrm{cm}$ ) at 2 , the 7 and 12 min time points. Following this, the activity of the SDH complex (pulled down by GFP) was determined by normalising changes in optical density over time $(\Delta \mathrm{OD} / 5 \mathrm{~min}$ and $\Delta \mathrm{OD} / 10 \mathrm{~min})$ with relative densitometry of SDHB-GFP eluted from the magnetic beads relative to the WT.

Published by Bioscientifica Ltd 


\section{Statistical analysis}

Experiments were performed in triplicate at least three times unless otherwise stated. Statistical analysis of the data was performed using ANOVA or repeated-measures ANOVA where appropriate. Differences between groups were evaluated by Fisher's protected least significant difference test and results were considered significant if $P<0.05$. The statistical package StatView for Windows version 5.0 (SAS Institute, Inc., Cary, NC, USA) was used. Results are expressed as mean \pm s.E.M. unless otherwise stated.

\section{Results}

\section{In silico structural modelling of SDHB mutations}

The human WT SDH model is shown in Fig. 1 and has a root mean square deviation of $0.18 \AA$ with the porcine SDH template for all atoms, or $0.12 \AA$ for backbone atoms. In silico analysis using only the models (hydrogen bond and electron path calculations, and visual inspection; refer to Table 1, Supplementary Table 1 and depictions in Supplementary Fig. 2, see section on supplementary data given at the end of this article) led to the identification of the following distinct types of mutations.

Truncation mutations As subunit B is structurally the central component of the SDH complex, the complex cannot be assembled when SDHB is significantly truncated or missing.

\section{Mutations disrupting macromolecular assem-} bly Loss of hydrogen bonding, loss of salt bridges or gain of repulsive charges between subunit B and subunits C or D may destabilise assembly of subunits C and D, leading to loss of electron pathways.

Mutations breaking electron pathways Loss of an iron-sulphur centre due to loss of bonding to the ironsulphur centre will result in lost electrons generating reactive oxygen species and failure to reduce ubiquinone.

Mutations affecting electron pathways The choreography of transferring two electrons from succinate to arrive in a stepwise sequence at ubiquinone may be perturbed in those mutants that have shortened or lengthened electron paths, resulting in generation of reactive oxygen species caused by lost electrons.

Mutations having no obvious consequences and located in a disease-associated mutation 'hot-spot' Some disease-associated mutations did not have obvious structurally deleterious effects and are located in a disease-associated mutation 'hot-spot' at the protein surface where a finger of subunit $C$ binds to the surface of subunit B (pictured in Supplementary Fig. 3, see section on supplementary data given at the end of this article). This leads us to speculate that this 'hot-spot' could be involved in binding of an assembly factor, such as an SDHB cofactor insertion or maturation factor (Van Vranken et al. 2014), whose binding to the SDH complex is compromised by these mutations.

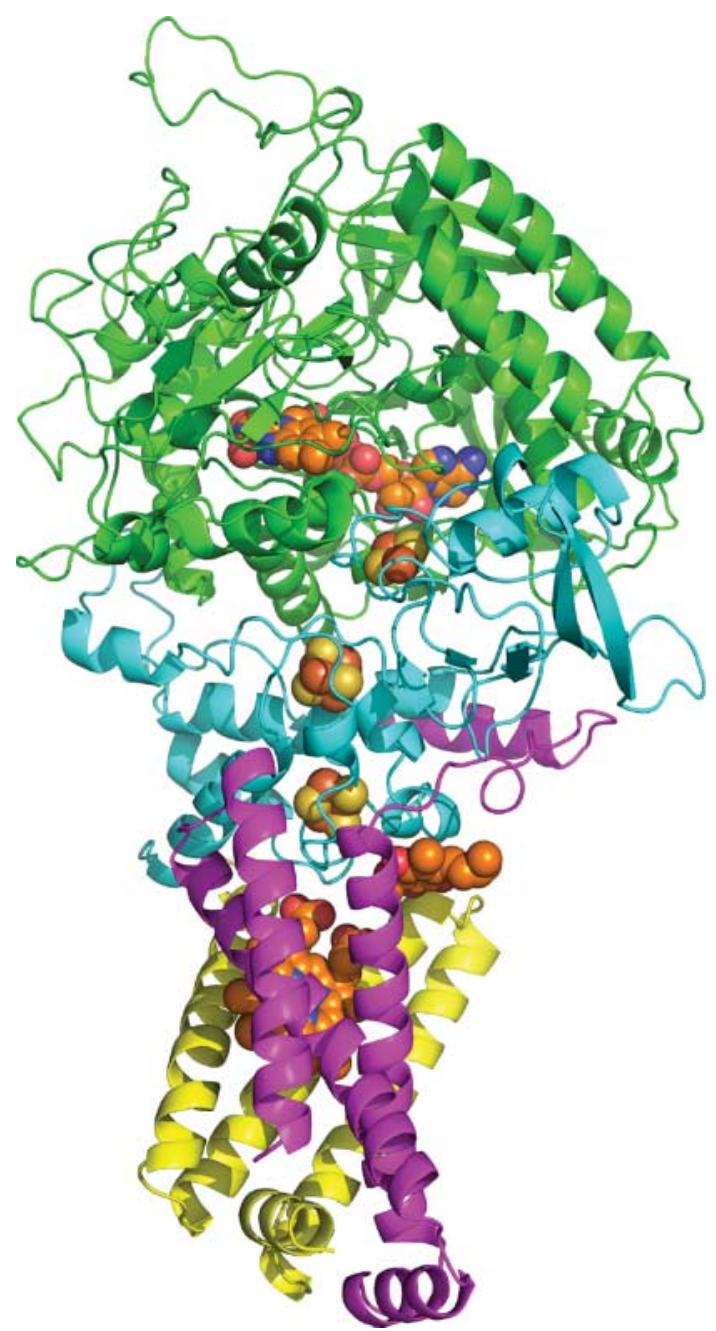

Figure 1

Human WT SDH homology model showing secondary protein structure (helices denote $\alpha$-helical secondary structure and ribbons denote $\beta$-sheets). Subunit A (green) is sitting on subunit B (cyan) and together they comprise the soluble component, which is sitting on subunits $C$ (magenta) and D (yellow) that are adjacent to each other in the membrane. Inside the complex is a path of redox factors (spheres, carbon atoms in orange) starting at $F A D$, passing through iron-sulphur centres and ending at ubiquinone. Other views of this model appear in Supplementary Fig. 1 and the locations of mutations studied are shown in Supplementary Figs 2 and 3.

Published by Bioscientifica Ltd 


\section{Impaired mitochondrial localisation of mutant SDHB in vitro is mutation-specific}

In order to validate these in silico predictions that $S D H B$ mutations could have diverse functional consequences, we then generated GFP-tagged SDHB constructs containing disease-associated mutations (Table 1). We chose mutations that, in our experience, have been associated with severe clinical manifestations (young age of onset, and/or malignant disease). The GFP-SDHB vectors were introduced into HEK293 cells, and initially studied for mitochondrial localisation. As expected, $S D H B$ truncating mutations (p.Arg27*, p.Arg90*, c.88delC and c.311delAinsGG) were not detected in whole-cell lysates (Fig. 2A) or in mitochondrial preparations (Fig. 2B). A strikingly different picture was seen with missense $S D H B$ mutations. All our missense mutant SDHB constructs (p.Ala43Pro, p.Arg46Gly, p.Arg46Gln, p.Ile127Ser, p.Pro197Arg, p.Cys101Tyr and p.Arg242His) were present in whole-cell lysates at levels similar to those for the WT SDHB construct (Fig. 2A). When mitochondrial-enriched preparations were analysed using anti-GFP western blots (and corrected for content of mitochondrial protein MT-CO2 as an internal control), some mutant SDHBs were present at concentrations not significantly different from those for the WT SDHB (p.Ile127Ser, p.Pro197Arg and p.Arg242His; Fig. 2B), whereas other mutant SDHBs exhibited markedly lower mitochondrial expression (p.Ala43Pro, p.Arg46Gly and p.Arg46Gln) $\left(n=4,{ }^{*} P<0.05\right)$.

\section{SDHB mutations differentially reduce SDH enzymatic activity}

The enzymatic function of SDH complexes containing only transfected SDHB (WT or mutant) was measured by means of anti-GFP antibody pulldown that specifically precipitated GFP-containing complexes while removing endogenous SDHB. Successful IP was confirmed by western blot (Fig. 3A). The rate of conversion from succinate to fumarate was then assessed in IPs by means of a modified Kirby protocol (Kirby et al. 2007), corrected for densitometric quantity of GFP-SDHB on western blot (relative to WT), and data are shown in Fig. 3B. No SDH activity was detected in IPs from cells transfected with GFP vector alone, nor in IPs from cells transfected with truncating $S D H B$ mutations p.Arg27*, p.Arg90*, c.88delC and c.311delAinsGG. Enzymatic activities of SDH complexes containing the missense SDHB mutants p.Ala43Pro, p.Arg46Gln and p.Arg242His were moderately reduced to approximately 50\% relative to WT (Fig. 3B).
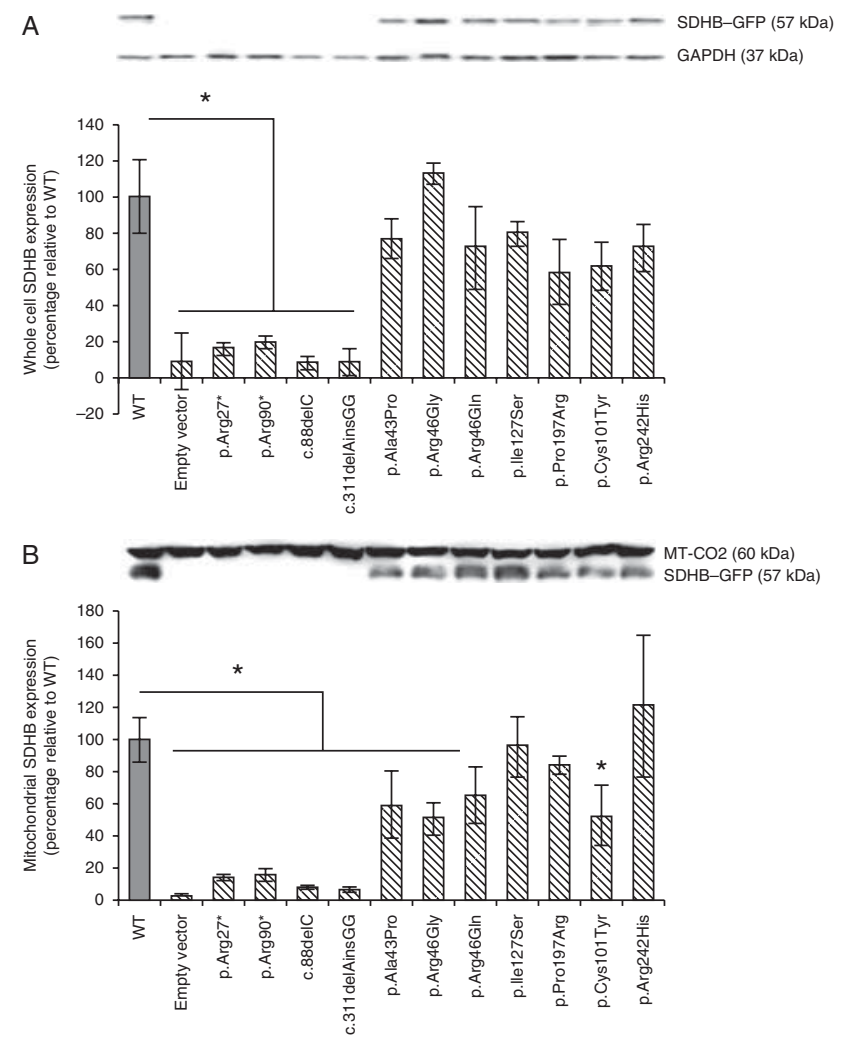

Figure 2

HEK293 cells were transiently transfected (24 h) with GFP-tagged SDHB (WT or mutants) or empty vector (pEGFP-N1) and the whole-cell fraction (A) and membrane fraction (B) were isolated. Samples were probed for GAPDH (whole-cell endogenous protein), MT-CO2 (mitochondrial membrane protein marker) and GFP on western blots. (A) GFP-tagged SDHB mutants p.Arg27*, p.Arg90*, c.88delC and c.311delAinsGG were not detected in the whole-cell fraction $\left({ }^{*} P<0.05\right.$, compared with WT), indicating nonsensemediated mRNA decay or protein degradation. (B) GFP-tagged $S D H B$ mutants p.Arg27*, p.Arg90*, c.88delC and c.311delAinsGG had baseline readings $\left({ }^{*} P<0.05\right)$. Additionally, compared with WT SDHB, SDHB mutants p.Ala43Pro, p.Arg46Gly and p.Arg46Gln, and p.Cys101Tyr showed significantly reduced localisation to mitochondria $\left({ }^{*} P<0.05\right)$. Results represent four independent experiments, with error bars indicating s.E.M., and ANOVA was used to test for statistical significance.

The p.Arg46Gly and p.Cys101Tyr mutants were found to be severely disruptive to SDH activity with the rate of metabolite conversion almost at baseline levels (Fig. 3B). Conversely, the reduction in SDH activity associated with either the p.Ile127Ser or the p.Pro197Arg mutation was not significantly different from that associated with the WT (Fig. 3B).

When the results of SDH assays were correlated with those from mitochondrial localisation, we found an excellent agreement for most mutations (Fig. 4). Notably, p.Arg242His was an obvious outlier with significantly reduced SDH activity despite near-normal mitochondrial abundance (Fig. 4).

Published by Bioscientifica Ltd. 

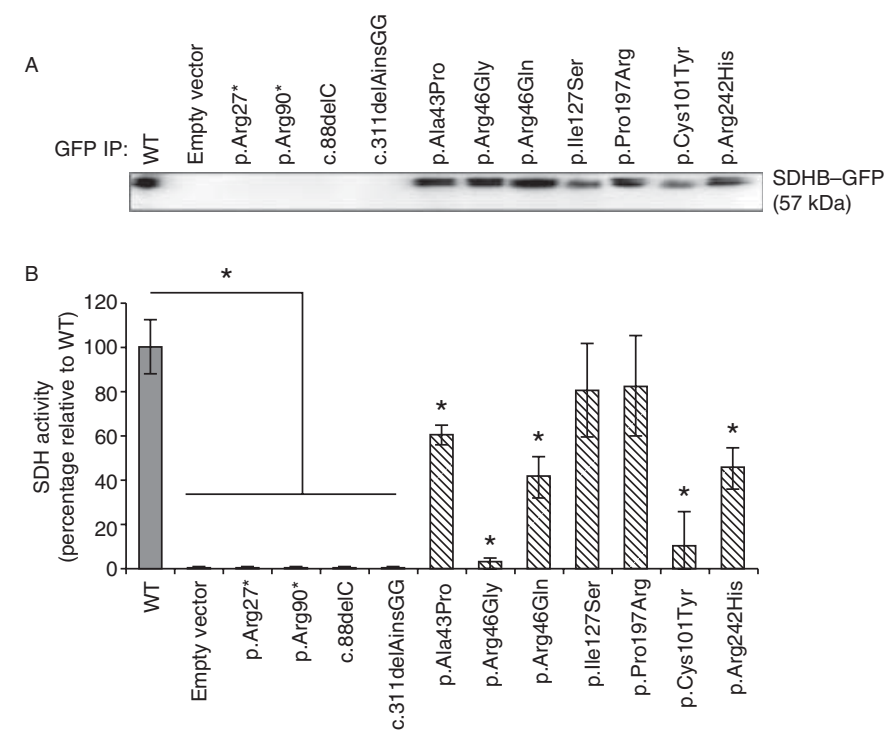

Figure 3

Activity of SDH isolated through pulldown by GFP-tagged SDHB. SDH complexes with transfected GFP-tagged SDHB in HEK293 cells were isolated through co-immunoprecipitation (IP) using magnetic beads. Enzyme activities were determined by measuring the reduction of the artificial electron acceptor DCPIP during conversion of succinate to fumarate using an absorbance assay (Kirby et al. 2007) over time, relative to WT and then normalised by pulled down SDHB-GFP western blotting. (A) Western blot of SDHB-GFP (WT and mutants) or empty vector (pEGFP-N1) after GFP IP. (B) GFP-tagged SDHB mutants, p.Arg27*, p.Arg90*, c.88delC and c.311delAinsGG, resulted in the absence of SDHB attached to GFP and SDH activities were not detected. While GFP-tagged SDHB mutants, p.Ala43Pro, p.Arg46GIn and p.Arg242His, significantly reduced SDH activity $\left({ }^{*} P<0.05\right)$, the greatest effect with respect to reduction of SDH activity was observed with p.Arg46Gly and p.Cys 101Tyr $\left({ }^{*} P<0.05\right)$. Results represent four independent experiments, with error bars indicating S.E.M., and ANOVA was used to test for statistical significance.

To assess whether tissue-specific factors may be important for determining mutation-specific SDH function, we repeated transfection studies of the same GFPSDHB vectors in the rat PC cell line PC12. SDH activity in IPs from these cells was strikingly similar to that in HEK293 cells (Supplementary Fig. 4, see section on supplementary data given at the end of this article).

\section{SDHB mutations differentially reduce association with SDHA}

SDHA is normally non-covalently associated with the SDHB subunit on the catalytic end of the SDH complex. The effects of $S D H B$ mutations on association between these two subunits were investigated by means of anti-GFP IP from whole-cell lysates, followed by western blotting with anti-SDHA (Fig. 5) and then correcting for densitometric quantity of GFP-SDHB (Fig. 5). As expected, IPs from cells transfected with truncating $S D H B$ mutations p.Arg27*, p.Arg90*, c.88delC and c.311delAinsGG did not express any SDHA (Fig. 5). SDHA was detected in IPs from cells transfected with missense $S D H B$ mutant constructs in amounts similar to those for GFP-WT SDHB IPs, except for p.Cys101Tyr that had significantly lower SDHA association $\left(n=4,{ }^{*} P<0.05\right)$.

\section{Discussion}

SDH dysfunction, arising as a result of mutations within any of the four SDH subunits, has been correlated with a range of diseases, including hereditary PC and PGL syndromes, renal cell carcinoma, gastrointestinal stromal tumours and Leigh syndrome (Horvath et al. 2006, Vicha et al. 2014). In general, PC and PGL tumours containing germline $S D H$ gene mutations exhibit markedly diminished SDH function (Gimenez-Roqueplo et al. 2002). Two predictable consequences of SDH inactivation are accumulation of succinate and increased production of reactive oxygen species, and both outcomes may contribute to the cellular accumulation of HIFs that is thought to mediate aberrant biological processes leading to tumour formation (Selak et al. 2005). However, functional consequences have not been precisely determined for most $S D H B$ mutations and this has fundamentally limited the ability to accurately correlate between genotype and phenotype in hereditary PGL syndrome type 4.

Herein, we used various techniques to investigate the functional outcomes of specific $S D H B$ mutations associated with hereditary PC and PGL. First, structural modelling of SDH was performed using X-ray crystallography data to predict the effect of mutations on protein stability, changes

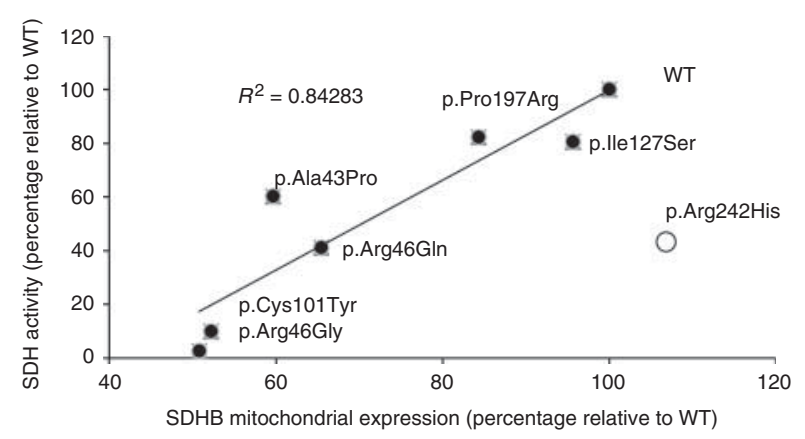

Figure 4

Correlation between mitochondrial expression of SDHB and SDH activity. Mean values (percentage relative to WT) of mitochondrial expression of GFP-tagged SDHB were plotted against mean values (percentage relative to WT) of SDH activity. A trendline encompassing investigated $S D H B$ mutants (excluding p.Arg242His) was added to indicate the direct relationship between SDH activity and SDHB mitochondrial localisation.

Published by Bioscientifica Ltd 


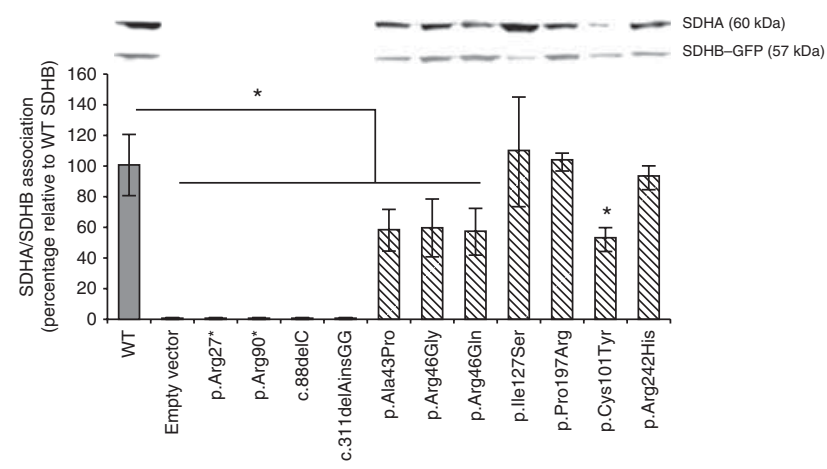

Figure 5

Association of mutant SDHB with WT SDHA. SDH complexes pulled down by GFP-tagged SDHB following $24 \mathrm{~h}$ of transfection in HEK293 cells were immunoblotted for SDHA and GFP. The p.Arg27*, p.Arg90*, c.88delC and c.311delAinsGG mutants show complete disassociation with SDHA due to absence of SDHB-GFP protein. Densitometry results of SDHA blots were normalised to SDHB-GFP proteins that were bound by the process of co-immunoprecipitation and showed that p.Ala43Pro, p.Arg46Gly, p.Arg46GIn and p.Cys101Tyr SDHB mutants significantly reduced the association between SDHB and SDHA ( $* P<0.05)$. Results represent four independent experiments, with error bars indicating S.E.M., and ANOVA was used to test for statistical significance.

in spatial dimensions affecting charges and binding sites in silico. These predictions were then compared against assessment of intracellular localisation and biochemical assays after transfecting HEK293 cells with various $S D H B$ mutants in vitro. We developed a novel means for assaying SDH function in human cells transfected with $S D H B$ mutant constructs, by specifically immunoprecipitating mutant-containing SDH complexes from whole cell lysates. Our results indicate that $S D H B$ mutations can result in three biological consequences: i) loss of protein due to nonsensemediated mRNA decay and/or accelerated degradation; ii) loss of correct mitochondrial targeting or iii) loss of enzyme function due to changes in specific active sites.

Structural modelling of disease-associated $S D H B$ mutations p.Arg27*, p.Arg90*, c.88delC and c.311delAinsGG predicted nonsense-mediated mRNA decay or truncation of the protein. Complete loss of SDHB expression in both cytosolic and mitochondrial compartments associated with truncating mutations from our in vitro data indicates either the occurrence of nonsensemediated decay or that the truncated proteins were unstable and degraded. In keeping with the localisation data, no measurable SDH activity was present in IPs containing these truncating mutants.

The second group of disease-associated $S D H B$ mutations (p.Ala43Pro, p.Arg46Gly and p.Arg46Gln) were those shown to exhibit reduced expression of mitochondrial SDHB despite being expressed at normal levels in cytoplasm. Again, in keeping with this localisation data, SDH activity was impaired by these mutations (but not totally abolished, as observed with the truncating mutations). Interestingly, p.Arg46Gly demonstrated severely impaired SDH activity, whereas p.Arg46Gln had a more modest effect on SDH function. These results indicate that these two different mutations have different effects on the SDH catalytic site.

The third group of disease-associated mutations (p.Cys101Tyr, p.Pro197Leu and p.Arg242His) were those associated with apparently normal mitochondrial SDHB abundance but impaired functional activity. Our structural modelling of these mutants led to the prediction of loss of the electron path (p.Cys101Tyr), changes to the electron path (p.Pro197Leu) or destabilisation of SDH structure leading to loss of the electron path (p.Arg242His). SDHB mutants p.Cys101Tyr and p.Arg242His were both associated with significantly reduced SDH activity when compared with the WT. Using our in silico results we predicted loss of bonding to the $2 \mathrm{Fe}-2 \mathrm{~S}$ centre at p.Cys 101 , which is more tolerant to amino acid substitution than the other two (4Fe-4S), (3Fe-4S) domains of SDHB (Werth et al. 1990, Iverson et al. 2012). Mutation at p.Arg242 was predicted to disrupt hydrogen bonds between SDH subunits, thereby reducing their biochemical efficiency and perhaps could also explain the loss of function in an otherwise fully compartmentalised SDH complex. The effect of p.Pro197Leu on measurable SDH activity in our assay was not significant, although our structural modelling predicts that this mutation has a clear effect on electron transport; it is intriguing to speculate that this mutation may actually discriminate between these two functions of the SDH complex.

In order to confirm that reduced expression of mitochondrial SDHB was in fact associated with loss of SDH assembly, we then examined the interaction of SDHB (WT or mutant) and SDHA. Our results were entirely consistent with the hypothesis that some mutations (p.Ala43Pro, p.Arg46Gly, p.Arg46Gln and p.Cys101Tyr) produce proteins that are normally stable but have impaired trafficking to mitochondria and/or assembly into mature SDH. The mechanism(s) by which this occurs remains speculative. Recent studies (Lane et al. 2014, Maio et al. 2014, Na et al. 2014) have highlighted the importance of iron-sulphur (Fe-S) clusters that are incorporated into $S D H B$ after engaging with two LYR motifs, including p.Arg46 and p.Arg242. This indicates

Published by Bioscientifica Ltd 
that mutation of these residues may cause failed LYRmediated assembly of Fe-S clusters.

Reports of two previous studies have described functional impairments associated with disease-associated SDHB mutations. Using transfection experiments in HeLa cells, Yang et al. (2012) proposed that the major effect of missense mutations was on protein stability, which we also found for some mutations (p.Ala43Pro and p.Arg46Gln). However, in contrast to Yang et al.'s (2012) suggestion that subcellular localisation and complex formation are not affected by $S D H B$ mutations, our results also convincingly indicated that some mutations either did not affect protein stability and instead selectively impaired SDH function (p.Arg242His), or had an effect on function that was disproportionate to the effect on mitochondrial SDHB expression (p.Cys101Tyr and p.Arg46Gly). In this respect, our findings were in agreement with the results of Panizza et al. (2013), who also found that SDHB mutants p.Arg46Leu, p.Cys101Tyr and p.Arg242Ser were severely impaired for SDH biochemical function in yeast. While Panizza et al. (2013) studied the biochemical function of SDHB mutants in $s d h 2$ null yeast cells, our novel approach of selectively immunoprecipitating mutant-containing SDH complexes allowed us to study the functional impact of these $S D H B$ mutations in human cells.

The results of this study have not enabled the identification of the biological effects of p.Ile127Ser. Structural modelling of this mutation did not identify an obvious pathological consequence. There was no clear effect on either mitochondrial SDH assembly or SDH activity, and the interaction between this mutant protein and SDHA appeared to be preserved. SDH activity was similarly preserved in p.Ile127Ser-containing IPs from PC12 cells. Despite this seemingly normal SDH function, in our experience, this mutation is recurrently associated with severe disease in multiple (unrelated) families. We therefore propose the hypothesis that p.Ile127Ser disrupts some aspects of SDHB function other than SDH activity (e.g. electron transport) and that further studies to precisely define pathogenic mechanisms for this mutant will be important.

Many studies (Gimenez-Roqueplo et al. 2001, 2002, Lima et al. 2007) have emphasised the importance of SDH structural integrity, and that specific mutations in any of the SDH subunits would disrupt assembly, anchorage to the mitochondrial membrane, surface exposure of binding sites and stabilisation of the complex. Our results indicate that particular $S D H B$ mutations may have specific effects on SDH structure and/or function as well as indicating a correlation between SDHB mitochondrial expression and
SDH activity (Fig. 4). Our results provide a platform upon which future genotype-phenotype correlations might be properly identified, by accurately characterising their mutational consequences. Furthermore, this study reveals new insights into the effects of $S D H B$ mutations and the power of structural modelling in predicting their biological consequences. In combination with recent metabolomics profiling (Richter et al. 2014), we predict that our functional assessment of $S D H B$ mutations will serve to better define specific consequences on SDH activity as well as to provide a much needed assay to distinguish pathogenic mutations from benign variants. In summary, pathogenic mutations of $S D H B$ have been shown to be associated with diverse structural and functional consequences broadly categorised into three groups: those that cause loss of SDHB protein, those that impair SDHB mitochondrial localisation and those that specifically disrupt enzyme sites.

\section{Supplementary data}

This is linked to the online version of the paper at http://dx.doi.org/10.1530/ ERC-15-0099.

\section{Declaration of interest}

The authors declare that there is no conflict of interest that could be perceived as prejudicing the impartiality of the research reported.

\section{Funding}

This work was supported by the Hillcrest Foundation (Perpetual Trustees) and the Pheo-Para Alliance.

\section{Author contribution statement}

E Kim conducted in vitro studies, performed data analyses and drafted the manuscript. V H M Tsang carried out selection and generation of SDHB expression vectors. E M Rath, A P Duff and W B Church carried out in silico studies of SDHB, providing predicted biological consequences as well as critically revising the manuscript. B G Robinson provided intellectual input and critically revised the manuscript. D E Benn participated in the design of the study, provided intellectual input and critically revised the manuscript. $T$ Dwight participated in the design of the study, provided intellectual input and critically revised the manuscript. R J Clifton-Bligh conceived, designed and coordinated the study and critically revised the manuscript.

\section{Acknowledgements}

The authors are grateful to Professor David Thorburn (Murdoch Childrens Research Institute, Melbourne) for advice on biochemical assays of SDH activity and to Dr Martyn Bullock (Kolling Institute, RNSH) for expert help with immunoprecipitation assays. They also thank our co-investigator Dr Kathy Tucker in the Australian SDH Consortium, and all the patients and families who are part of this ongoing study.

Published by Bioscientifica Ltd 


\section{References}

Adzhubei IA, Schmidt S, Peshkin L, Ramensky VE, Gerasimova A, Bork P, Kondrashov AS \& Sunyaev SR 2010 A method of server for predicting damaging missense mutations. Nature Methods 7 248-249. (doi:10.1038/nmeth0410-248)

Amar L, Bertherat J, Baudin E, Ajzenberg C, Paillerets B, Chabre O, Chamontin B, Delemer B, Giraud S, Murat A et al. 2005 Genetic testing in pheochromocytoma or functional paraganglioma. Journal of Clinical Oncology 23 8812-8818. (doi:10.1200/JCO.2005.03.1484)

Balabin IA, Hu X \& Beratan DN 2012 Exploring biological electron transfer pathway dynamics with the Pathways plugin for VMD. Journal of Computational Chemistry 33 906-910. (doi:10.1002/jcc.22927)

Barrientos A 2003 Yeast models of human mitochondrial diseases. International Union of Biochemistry and Molecular Biology 55 83-95. (doi:10.1002/tbmb.718540876)

Bayley J, Devilee P \& Taschner PE 2005 The SDH mutation database: an online resource for succinate dehydrogenase sequence variants involved in pheochromocytoma, paraganglioma and mitochondrial complex II deficiency. BMC Medical Genetics 639-45. (doi:10.1186/1471-2350-6-39)

Benn DE, Gimenez-Roqueplo AP, Reilly JR, Bertherat J, Burgess J, Byth K, Croxson M, Dahia PL, Elston M, Gimm O et al. 2006 Clinical presentation and penetrance of pheochromocytoma/paraganglioma syndromes. Journal of Clinical Endocrinology and Metabolism 91 827-836. (doi:10.1210/jc.2005-1862)

Beratan DN, Betts JN \& Onuchic JN 1991 Protein electron transfer rates set by the bridging secondary and tertiary structure. Science $\mathbf{2 5 2}$ 1285-1288. (doi:10.1126/science.1656523)

Berman H, Henrick K \& Nakamura H 2000 Announcing the worldwide Protein Data Bank. Nature Structural \& Molecular Biology 10980. (doi:10.1038/nsb1203-980)

Castro-Vega LJ, Buffet A, De Cubas AA, Cascon A, Menara M, Khalifa E, Amar L, Azriel S, Bourdeau I, Chabre O et al. 2014 Germline mutations in $F H$ confer predisposition to malignant pheochromocytomas and paragangliomas. Human Molecular Genetics 23 2440-2446. (doi:10.1093/hmg/ddt639)

Dahia PL 2014 Pheochromocytoma and paraganglioma pathogenesis: learning from genetic heterogeneity. Nature Reviews. Cancer $\mathbf{1 4}$ 108-119. (doi:10.1038/nrc3648)

Dahia PL, Ross KN, Wright ME, Hayashida CY, Santagata S, Barontini M, Kung AL, Sanso G, Powers JF, Tischler AS et al. 2005 A HIF1 $\alpha$ regulatory loop links hypoxia and mitochondrial signals in pheochromocytomas. PLoS Genetics 1 72-80. (doi:10.1371/journal.pgen.0010008)

DeLano WL 2002 The PyMOL Molecular Graphics System (DeLano Scientific LLC, San Carlos, CA, USA, http://www.pymol.org).

Denko NC 2008 Hypoxia, HIF1 and glucose metabolism in the solid tumour. Nature Reviews. Cancer 8 705-713. (doi:10.1038/nrc2468)

Dwight T, Benn DE, Clarkson A, Vilain R, Lipton L, Robinson BG, CliftonBligh RJ \& Gill AJ 2013 Loss of SDHA expression identifies SDHA mutations in succinate dehydrogenase-deficient gastrointestinal stromal tumors. American Journal of Surgical Pathology 37 226-233. (doi:10.1097/PAS.0b013e3182671155)

Eswar N, Webb B, Marti-Remon MA, Madhusudhan MS, Eramian D, Shen MY, Pieper U \& Sali A 2006 Comparative protein structure modeling using Modeller. Current Protocols in Bioinformatics 15 5.6.1-5.6.30. (doi:10.1002/0471250953.bi0506s15)

Fokkema IF, Taschner PE, Schaafsma GC, Celli J, Laros JF \& den Dunnen JT 2011 LOVD v.2.0: the next generation in gene variant databases. Human Mutation 32 557-563. (doi:10.1002/humu.21438)

Gill AJ, Chou A, Vilain R, Clarkson A, Lui M, Jin R, Tobias V, Samra J, Goldstein D, Smith RC et al. 2010 Immunohistochemistry for SDHB divides gastrointestinal stromal tumors (GISTs) into 2 distinct types. American Journal of Surgical Pathology 34 636-644. (doi:10.1097/PAS. Ob013e3181d6150d)

Gill AJ, Pachter NS, Chou A, Young B, Clarkson A, Tucker KM, Winship IM, Earls P, Benn DE, Robinson BG et al. 2011 Renal tumors associated with germline SDHB mutation show distinctive morphology. American Journal of Surgical Pathology 35 1578-1585. (doi:10.1097/PAS. Ob013e318227e7f4)

Gimenez-Roqueplo AP, Favier J, Rustin P, Mourad J, Plouin P, Corvol P, Rotig A \& Jeunemaitre X 2001 The R22X mutation of the SDHD gene in hereditary paraganglioma abolishes the enzymatic activity of complex II in the mitochondrial respiratory chain and activates the hypoxia pathway. American Journal of Human Genetics 69 1186-1197. (doi:10. 1086/324413)

Gimenez-Roqueplo AP, Favier J, Rustin P, Mourad JJ, Plouin PF, Corvol P, Rötig A \& Jeunemaitre X 2002 Functional consequences of a $S D H B$ gene mutation in an apparently sporadic pheochromocytoma. Journal of Clinical Endocrinology and Metabolism 87 4771-4774. (doi:10.1210/jc. 2002-020525)

Gimenez-Roqueplo AP, Dahia PL \& Robledo M 2012 An update on the genetics of paraganglioma, pheochromocytoma, and associated hereditary syndromes. Hormone and Metabolic Research 44 328-333. (doi:10.1055/s-0031-1301302)

Goffrini P, Ercolino T, Panizza E, Giachè V, Cavone L, Chiarugi A, Dima V, Ferror I \& Mannelli M 2009 Functional study in a yeast model of a novel succinate dehydrogenase subunit $B$ gene germline missense mutation (C191Y) diagnosed in a patient affected by a glomus tumor. Human Molecular Genetics 18 1860-1868. (doi:10.1093/hmg/ddp102)

Horvath R, Abicht A, Holinski-Feder E, Laner A, Gempel K, Prokisch H \& Jaksch M 2006 Leigh syndrome caused by mutations in the flavoprotein (Fp) subunit of succinate dehydrogenase (SDHA). Journal of Neurology, Neurosurgery, and Psychiatry 77 74-76. (doi:10.1136/jnnp.2005.067041)

Humphrey W, Dalke A \& Schulten K 1996 VMD: visual molecular dynamics. Journal of Molecular Graphics 14 33-38. (doi:10.1016/ 0263-7855(96)00018-5)

Imperiale A, Moussallieh FM, Sebag F, Brunaud L, Barlier A, Elbayed K, Bachellier P, Goichot B, Pacak K, Namer IJ et al. 2013 A new specific succinate-glutamate metabolomic hallmark in Sdhx-related paragangliomas. PLoS ONE 8 e80539. (doi:10.1371/journal.pone.0080539)

Iverson TM, Maklashina E \& Cecchini G 2012 Structural basis for malfunction in complex II. Journal of Biological Chemistry $\mathbf{2 8 7}$ 35430-35438. (doi:10.1074/jbc.R112.408419)

Jochmanová I, Zelinka T, Widimský J \& Pacak K 2014 HIF signaling pathway in pheochromocytoma and other neuroendocrine tumors. Physiological Research 63 S251-S262.

Kirby DM, Thorburn DR, Turnbull DM \& Taylor RW 2007 Biochemical assays of respiratory chain complex activity. Methods in Cell Biology 80 93-119. (doi:10.1016/S0091-679X(06)80004-X)

Lane DJ, Merlot AM \& Richardson DR 2014 The lure of a LYR: the logistics of iron sulfur cluster delivery. Cell Metabolism 19 348-350. (doi:10.1016/j.cmet.2014.02.011)

Lenders J, Duh QY, Eisenhofer G, Gimenez-Roqueplo AP, Grebe S, Murad MH, Naruse M, Pacak K \& Young W 2014 Pheochromocytoma and paraganglioma: an endocrine clnincal practice guideline. Journal of Clinical Endocrinology and Metabolism 99 1915-1945. (doi:10.1210/jc. 2014-1498)

Lima J, Feijao T, Ferreira da Silva A, Pereira-Castro I, Fernandez-Ballester G, Máximo V \& Garcia-Rostan G 2007 High frequency of germline succinate dehydrogenase mutations in sporadic cervical paragangliomas in northern Spain: mitochondrial succinate dehydrogenase structure-function relationships and clinical-pathological correlations. Journal of Clinical Endocrinology and Metabolism 92 4853-4864. (doi:10.1210/jc.2007-0640)

Lorenzo FR, Yang C, Ng Tang Fui M, Vankayalapati H, Zhuang Z, Huynh T, Grossmann M, Pacak K \& Prchal JT 2013 A novel EPAS1/HIF2A germline mutation in a congenital polycythemia with paraganglioma. Journal of Molecular Medicine 91 507-512. (doi:10.1007/s00109-012-0967-z)

Maio N, Singh A, Uhrigshardt H, Saxena N, Tong WH \& Rouault TA 2014 Cochaperone binding to LYR motifs confers specificity of iron sulfur cluster delivery. Cell Metabolism 19 445-457. (doi:10.1016/j.cmet.2014. 01.015) 
McDonald IK \& Thornton JM 1994 Satisfying hydrogen bonding potential in proteins. Journal of Molecular Biology 238 777-793. (doi:10.1006/ jmbi.1994.1334)

Na U, Yu W, Cox J, Bricker DK, Brockmann K, Rutter J, Thummel CS \& Winge DR 2014 The LYR factors SDHAF1 and SDHAF3 mediate maturation of the iron-sulfur subunit of succinate dehydrogenase. Cell Metabolism 20 253-266. (doi:10.1016/j.cmet.2014.05.014)

Ng PC \& Henikoff S 2001 Predicting deleterious amino acid substitutions. Genome Research 11 863-874. (doi:10.1101/gr.176601)

Panizza E, Ercolino T, Mori L, Rapizzi E, Castellano M, Opocher G, Ferrero I, Neumann HP, Mannelli M \& Goffrini P 2013 Yeast model for evaluating the pathogenic significance of $S D H B, S D H C$ and $S D H D$ mutations in PHEO-PGL syndrome. Human Molecular Genetics 22 804-815. (doi:10.1093/hmg/dds487)

Poliakov E, Managadze D \& Rogozin I 2014 Generalized portrait of cancer metabolic pathways inferred from a list of genes overexpressed in cancer. Genetics Research International 646193 1-8. (doi:10.1155/2014/646193)

Richter S, Peitzsch M, Rapizzi E, Lenders J, Qin N, de Cubas A \& Eisenhofer G 2014 Krebs cycle metabolite profiling for identification and stratification of pheochromocytomas/paragangliomas due to succinate dehydrogenase deficiency. Journal of Clinical Endocrinology and Metabolism 99 3903-3911. (doi:10.1210/jc.2014-2151)

Šali A \& Blundell TL 1993 Comparative protein modelling by satisfaction of spatial restraints. Journal of Molecular Biology 234 779-815. (doi:10.1006/jmbi.1993.1626)

Selak MA, Armour SM, Mackenzie ED, Boulahbel H, Watson DG, Mansfield KD, Pan Y, Simon MC, Thompson CB \& Gottlieb E 2005 Succinate links TCA cycle dysfunction to oncogenesis by inhibiting HIF- $\alpha$ prolyl hydroxylase. Cancer Cell 7 77-85. (doi:10.1016/j.ccr.2004.11.022)
Srirangalingam U, Walker L, Khoo B, MacDonald F, Gardner D, Wilkin T, Skelly R, George E, Spponer D, Chew SL et al. 2008 Clinical manifestations of familial paraganglioma and phaeochromocytomas in succinate dehydrogenase B (SDH-B) gene mutation carriers. Clinical Endocrinology 69 587-596. (doi:10.1111/j.1365-2265.2008.03274.x)

Sun F, Huo X, Zhai Y, Wang A, Xu J, Su D, Bartlam M \& Rao Z 2005 Crystal structure of mitochondrial respiratory membrane protein complex II. Cell 121 1043-1057. (doi:10.1016/j.cell.2005.05.025)

Van Vranken JG, Na U, Winge DR \& Rutter J 2014 Protein-mediated assembly of succinate dehydrogenase and its cofactors. Critical Reviews in Biochemistry and Molecular Biology 9 1-13. (doi:10.3109/10409238. 2014.990556)

Vicha A, Taieb D \& Pacak K 2014 Current views on cell metabolism in SDHx-related pheochromocytoma and paraganglioma. EndocrineRelated Cancer 21 261-277. (doi:10.1530/ERC-13-0398)

Wallace AC, Laskowski RA \& Thornton JM 1995 LIGPLOT: a program to generate schematic diagrams of protein-ligand interactions. Protein Engineering, Design \& Selection 8 127-134. (doi:10.1093/protein/ 8.2.127)

Werth MT, Cecchini G, Manodori A, Ackrell BA, Schröder I, Gunsalus RP \& Johnson MK 1990 Site-directed mutagenesis of conserved cysteine residues in Escherichia coli fumarate reductase: modification of the spectroscopic and electrochemical properties of the [2Fe-2S] cluster. PNAS 87 8965-8969. (doi:10.1073/pnas.87.22.8965)

Yang C, Matro JC, Huntoon KM, Donald YY, Huynh YT, Fliedner SM, Breza J, Zhuang Z \& Pacak K 2012 Missense mutations in the human SDHB gene increase protein degradation without altering intrinsic enzymatic function. FASEB Journal 26 4506-4516. (doi:10.1096/ fj.12-210146)

Received in final form 2 March 2015

Accepted 23 March 2015
(C) 2015 Society for Endocrinology Printed in Great Britain 